journal club

\title{
Erneute Antikoagulation nach Hirnblutung ist prognostisch sinnvoll
}

Fragestellung: Die Studie untersuchte die Wirksamkeit und das Risiko einer erneuten Antikoagulation nach einer intrakraniellen Blutung.

Hintergrund: Die häufigste Indikation für eine orale Antikoagulation ist das Vorhofflimmern. In diesem Zusammenhang kann das Schlaganfallrisiko um 60 - 70 \% reduziert werden. Die am meisten gefürchtete Komplikation der oralen Antikoagulation ist die intrazerebrale Blutung mit einer Sterblichkeit von bis $\mathrm{zu} 45 \%$.

Bisher gibt es nur wenige Studien, die untersucht haben, ob es möglich ist, bei Patienten mit abgelaufener intrakranieller Blutung und Vorhofflimmern eine erneute Antikoagulation durchzuführen. Die vorliegende Metaanalyse fasst die bisherigen Kohortenstudien zu dieser Fragestellung zusammen.

Patienten und Methodik: Es handelt sich um eine systematische Literaturrecherche. Erfasst wurden Studien, die Patienten untersuchten, die unter Antikoagulanzien eine intrakranielle Blutung erlitten, und ob diese Antikoagulation zu einem späteren Zeitpunkt wiederholt wurde. Endpunkte waren thromboembolische Ereignisse (ischämischer Schlaganfall und Myokardinfarkt) und erneute intrakranielle Blutungen. AnschlieBend wurde eine Metaanaly-

Murthy SB, Gupta A, Merkler $\mathrm{AE}$ et al. Restarting anticoagulant therapy after intracranial hemorrhage: A systematic review and meta-analysis. Stroke 2017; 48: doi 10.11.61. se durchgeführt.

Ergebnisse: Es konnten acht Studien mit insgesamt 5.306 Patienten mit intrakraniellen Blutungen ausgewertet werden. Vier der Studien schlos- sen nur Patienten mit parenchymatösen Hirnblutungen ein, die übrigen auch Patienten mit Subduralhämatomen und Subarachnoidalblutungen. Die häufigste Indikation für die orale Antikoagulation war Vorhofflimmern, gefolgt von mechanischen Herzklappen, venösen Thromboembolien und vorausgegangenen Schlaganfällen. Wenn die Antikoagulation erneut durchgeführt wurde, geschah dies zwischen den Tagen 10 und 39. Bei sieben der acht Studien erfolgte die orale Antikoagulation mit Vitamin-K-Antagonisten.

Für die Beziehung zwischen erneuter Antikoagulation und thromboembolischen Komplikationen standen sechs Studien mit 2.044 Patienten zur Verfügung. In diesen sechs Studien wurde die Antikoagulation bei 786 Patienten wieder aufgenommen. Dies entspricht einer Rate von 38,4\%. Bei den Personen, die erneut antikoaguliert wurden, lag die Häufigkeit thromboembolischer Ereignisse bei 6,7\%, im Vergleich zu 17,6\% bei Patienten, die nicht erneut antikoaguliert werden konnten.

Acht Studien konnten bezüglich des Risikos einer erneuten intrakraniellen Blutung ausgewertet werden. Die Auswertung umfasste 5.306 Patienten, von denen 1.899 - entsprechend $35,8 \%$ - erneut antikoaguliert wurden. Eine erneute intrakranielle Blutung wurde bei $8,7 \%$ der Patienten beobachtet, die erneut antikoaguliert wurden, und bei 7,8\%, die nicht erneut antikoaguliert wurden.

Schlussfolgerungen: In Kohortenstudien haben Patienten, die unter einer oralen Antikoagulation eine intrakranielle Blutung erlitten haben, eine bessere Prognose bezüglich erneuter thromboembolischer Ereignisse, wenn es möglich ist, die Antikoagulation zu wiederholen.

\section{- Kommentar von Hans-Christoph Diener, Essen}

\section{Die endgültige Antwort kann nur eine randomisierte Studie geben}

Diese Literaturrecherche und Metaanalyse ist hilfreich, da sie zeigt, dass es möglich ist, bei bis zu $40 \%$ aller Patienten, die unter Antikoagulanzien eine intrakranielle Blutung erlitten haben, die Antikoagulation zu einem späteren Zeitpunkt zu wiederholen. Wenn dies mit Blick auf das Blutungsrisiko möglich ist, haben diese Patienten einen eindeutigen Nutzen bezüglich der Verhinderung weiterer thromboembolischer Ereignisse.

Die Kohortenstudien sind natürlich nicht randomisiert, sodass eine erhebliche Verfälschung der Ergebnisse zu erwarten ist. Bei $60 \%$ der Patienten waren die behandelnden Ärzte offenbar der Meinung, dass das Risiko einer erneuten Antikoagulation bezüglich einer erneuten intrakraniellen Blutung zu hoch wäre. Die Frage, ob und wann nach stattgehabter intrakranieller Blutung erneut antikoaguliert werden kann, kann nur durch eine sorgfältige geplante und durchgeführte randomisierte Studie beantwortet werden.

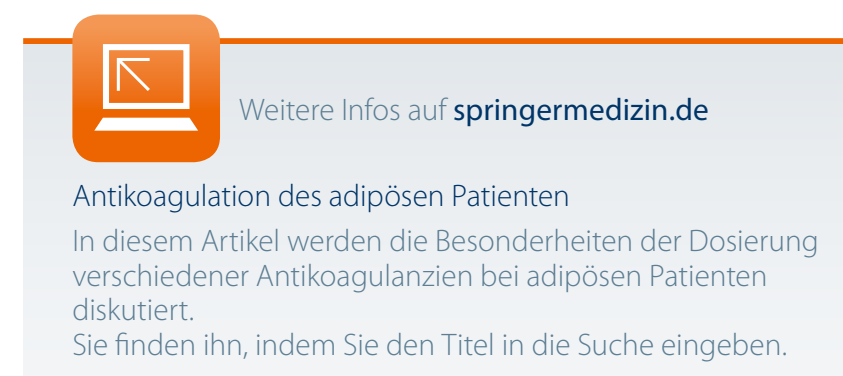

